\title{
APRENDIZAGEM COLABORATIVA: ATIVIDADES DE GRUPO COMO NÚCLEO E USO DO COMPUTADOR COMO CONTEXTO
}

\author{
Collaborative Learning: Group Activities as Nucleus and \\ Computer Support as Context
}

Juracy C. Marques ${ }^{1}$

\section{Resumo}

Discutem-se algumas teorias de aprendizagem que contribuem para o melhor entendimento da Aprendizagem Colaborativa centrada nas atividades de grupo em seus processos de interação e interatividade virtual. Destaca-se a importância de ambientes de aprendizagem que ensejem uma perspectiva que acolha à mediação de dispositivos culturais nas atividades de grupo e na aprendizagem, pelo diálogo. $\mathrm{O}$ uso do computador como contexto preferencial das ações das pessoas e dos grupos que correspondam às suas motivações, objetivos e interesses, sabendo-se que o pensamento e o raciocínio estão sempre imbuídos do que ocorre no plano social. Os participantes são marcados pela mediação de seus instrumentos culturais, o que os torna mais flexíveis para testar e comunicar suas idéias, organizando suas atividades e os conseqüentes resultados de aprendizagem. As mediações são interdependentes, mas podem ser analisadas em diferentes níveis, as condições concretas, o refinamento das idéias ao contrastar as suas com as dos outros em face das experiências vividas, alterando seus pressupostos e visões de mundo. Defende-se que o mero uso do computador ou da Intemet com seus múltiplos equipamentos não se constituem, por si, numa mudança teórica sobre o que é aprender, pode ser mera repetição de antigas concepções e teorias de aprendizagem revestidas pelo charme do impacto dos recursos de multimídia. Questiona-se o conhecimento sobre como ocorrem as mediações sociais e culturais nos processos de aprendizagem em diferentes estruturas de participação e de como os instrumentos culturais tornam-se mediadores da cognição e das atividades de grupo, sugerindo-se que isso pode vir a ser uma agenda para futuras pesquisas.

Palavras-chave: Mediação; Cultura; Uso do computador; Teorias de aprendizagem.

1 Psicóloga. Ex-Coordenadora de Grupos de Pesquisa em Educação, UFRGS e em Psicologia, PUCRS. Endereço para contato: Rua Engenheiro Olavo Nunes, 237, ap. 502. Porto Alegre - RS. 90440-170. E-mail: juracycm@brturbo.com.br 
Abstract

Theories of learning that contribute to the meaning of Collaborative Leaming as the center of group activities in its interaction and virtual interativity are revised and discussed. The importance of leaming environments planning, inside a perspective of acceptance of cultural artifacts as mediators for learning through activity in its criative dialogue are pointed out. The computer support as a preferential context for people and group actions, according to its motivations, aims e purposes are discussed, enlightened by the notion that cognition and reasoning have their origin in social contexts. Participants are heavily dependent on the material mediation of cultural tools, which enable them to be more flexible to test their ideas and to communicate with each other to organize their activities and the learning outcomes as well. There are interdependencies of mediation, though it can be analized in different levels, the concrete conditions, the refinement of ideas based on the inputs of others, connecting them with the lived experiences, altering previous assumptions and world views. However, the simple use of computer support to impart knowledge doesn't change by itself the theoretical understanding of what is learning, it might be a repetition of the old theories dressed up with the charming of multimedia impact. How cultural tools mediate cognition and group activities by social interactions under different structures of participation are questionned, as possible agend for the advancement of research.

Keywords: Mediation; Culture; Computer support; Learning theories.

\section{Introdução}

Aprendizagem colaborativa pode ser construída e alcançada num ambiente de aprendizagem que tem como suporte o auxílio do computador. Entretanto, isso em si não basta, pois os resultados de aprendizagem dependem muito mais das teorias nas quais nos apoiamos do que dos recursos tecnológicos que utilizamos. Quais são essas teorias?

(1) Toda atividade de aprendizagem é mediada por dispositivos culturais que estimulam, informam e sustentam os propósitos para os quais essa atividade é oferecida, conforme o desenho de uma situação que aponta para determinados objetivos ou solução de problemas relevantes.

(2) A atividade é planejada e fundamentada em conceitos e noções bem definidos que se apresentam em vários e diferentes níveis que, todavia, são interdependentes entre si, reforçandose no encaminhamento de elaborações cada vez mais complexas.

(3) A compreensão das noções, princípios e conceitos que vão sendo aprendidos originam-se do que está socialmente estabelecido naquela particular comunidade e, por isso, raramente, produz uma reação de estranhamento quando emerge na própria atividade que está sendo desenvolvida.

A perspectiva teórica é de que a aprendizagem centra-se nas atividades e não no professor ou no aluno ou mesmo na interação entre eles (Gifford, B. R., Enyedy, N.D., 1999). Assim, a apren- dizagem é entendida como um processo complexo em que as cognições individuais se fazem a partir das condições materiais oferecidas e das formas de participação ensejadas pelo ambiente que as cria, de conformidade com essas mesmas participações.

Desse modo, recusa-se a posição de que o conhecimento é produzido a partir de um objeto no qual uma pessoa possui conhecimento que pode ser aplicado em todo e qualquer contexto, que passa da mente do professor para a mente do estudante, sem nenhum compromisso com relevância, implicações e conseqüências. Nesse caso, a aprendizagem será tanto mais eficiente quanto mais o foco for bem delimitado, podendo mover-se indefinidamente de uma situação para outra, sem desvirtuamento de seu núcleo de verdade (Gifford, 1999).

\section{Teorias de Aprendizagem}

Temos múltiplas propostas pedagógicas nas quais o ensino é centrado no professor. Não importa se o professor é diretamente substituído pelo computador, com os inerentes vícios e virtudes do ensino tradicional. $\mathrm{O}$ fundamento de tais posturas é a de que o professor (ou o computador) possui o conhecimento que deve ser transferido para 0 estudante. Nesse modo de ensinar, a órbita em que se movimentam as atividades de aula se desenvolvem sob a única e exclusiva orientação do professor ou do computador, como 
fonte da qual emana o conhecimento, a informação e coerentemente a avaliação do processo e dos resultados de aprendizagem. A tecnologia torna-se um recurso para enfatizar essa perspectiva, produzindo aulas expositivas atraentes e persuasivas, revestidas de recursos de multimídia que, sem dúvida, aumentam suas potencialidades, como instrumento para alcançar os objetivos de aprendizagem. Entretanto, as teorias de aprendizagem por trás de tais procedimentos são as mesmas, não alterando as características básicas deste sistema de ensino. O ponto crítico reside na ausência de compreensão do contexto social em que tais aprendizagens são produzidas, tendo como referência o pressuposto de que a aprendizagem é, sobretudo, uma atividade individual (Lave, 1996).

A questão crítica é o quanto e quando a aprendizagem é individual ou demonstrar o quanto e como ela é resultante, em larga medida, de ações colaborativas que empregam diferentes tipos de inteligência, para além do estreito conceito de QI (quociente intelectual) (Marques, 2004; Sternberg, 1985). Nesta ótica, o raciocínio lógico é, apenas, uma das dimensões - a criatividade, a inteligência prática e a sabedoria, segundo Sternberg, (1985) - que somente se revestirá de eficiência e eficácia, quando combinada com outras habilidades e competências exigidas pelo contexto em que aquela situação de aprendizagem é posta a exame e análise, naquele ambiente específico de aprendizagem. Contudo, as posições individualistas tendem a ignorar o papel da conversação entre os pares, como parte integrante dos processos de aprendizagem e como um dos instrumentos de mediação das estruturas sociais e culturais.

Quando a aprendizagem é centrada no aluno, minimiza-se a importância do contexto social, bem como o papel decisivo da conversação e da colaboração como partes determinantes dos processos de aprendizagem. Não se levam em conta as múltiplas aprendizagens que ocorrem de maneira clara ou sutil por influência dos colegas, das dinâmicas ensejadas por meio dos trabalhos de grupo dos alunos, nem de suas motivações, ao interpretar os significados de elementos sugestivos nas situações vividas, por meio de intercâmbio de experiências e das relações afetivas que as perpassam. Deixa-se de reconhecer os efeitos de reflexões ensejadas no nível intelectual que busca significados nas inter-relações das variáveis implicadas pelas atividades que demandam criatividade e pensamento reflexivo, propiciando insights sobre fatos e verdades até então desconhecidos. Falta conexão do que está sendo vivido com as experiências passadas, portanto, com a bagagem de conhecimento que cada estudante traz para a atividade em desenvolvimento que é, sem dúvida, portadora da cultura em que estão imersos.

\section{Figura 1 - Atividade como núcleo de aprendizagem}

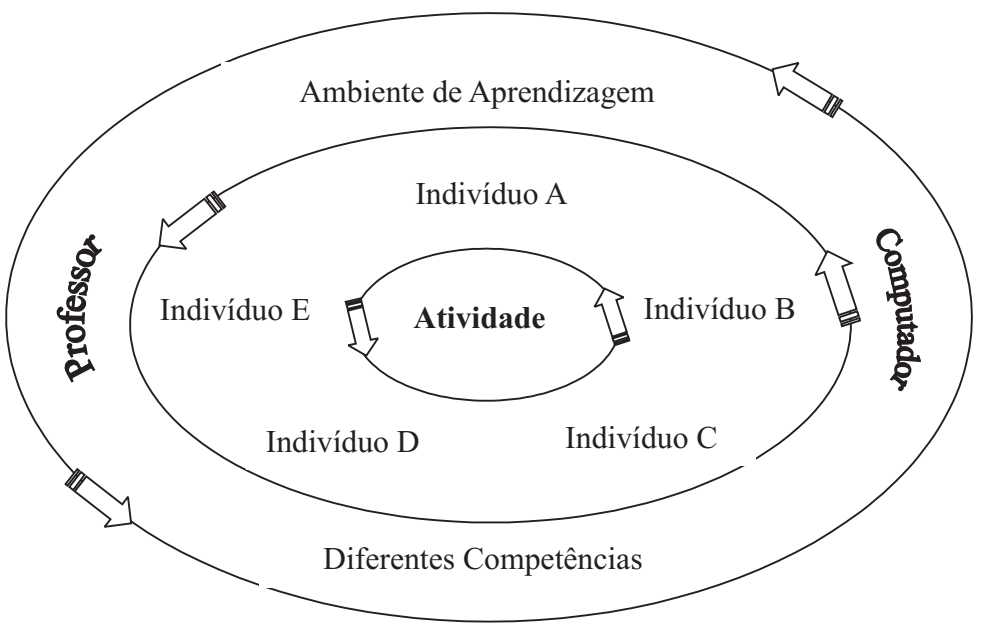

Psicologia Argumento, Curitiba, v. 24, n. 44 p. 37-43, jan./mar. 2006. 
Schwandt (1999) distingue os conceitos de conhecer e compreender, defendendo que toda compreensão é um processo de aprendizagem relacional, que traz em seu íntimo a possibilidade de incompreensão, pois as situações nas quais nos engajamos não são independentes de nossas interpretações. Por conseguinte, a aprendizagem, a mudança de ótica, traz uma marca de opacidade que pode ser esclarecida pelas atividades de reflexão em grupo, atravessadas pelas múltiplas visões que ali são embaralhadas, a fim de serem re-ordenadas, tendo em vista os objetivos e as motivações que animam as discussões (Marques, 2004, p. 18).

Em vez de focar as aprendizagens individuais, a aprendizagem colaborativa cria um sistema de aprendizagem em que o centro de atenções, processos interativos, feedbacks e avaliações produzem-se em conseqüência das atividades dos grupos de alunos que se ligam e se fundam como células de aprendizagem. Mas ainda aqui há o perigo de repetir as mesmas persistentes teorias individualistas de como se aprende, sem alterar a estrutura ou o modelo de ensinar. O grupo, então, torna-se o centro do ensino, usando o professor (ou o computador) como um recurso de aprendizagem, mas sem incorporar as múltiplas variáveis que estão na órbita do contexto, incluindo a subjetividade e as trocas de experiências que emergem das exigências das atividades em desenvolvimento.

Em conseqüência, a aprendizagem é fundamentalmente social, realizando-se, entretanto, na experiência de todos e de cada um mediante a utilização dos recursos que configuram os inter-relacionamentos entre os conteúdos e o contexto que os dinamiza. Os cursos planejados para incluir uso de computador facilitam o desenvolvimento de atividades que se atravessam com fontes diversificadas, reforçando os focos de interesse e os valores da cultura de que estão impregnados. Cria-se uma história compartilhada com nítidas identificações com os valores e significados relevantes para aquele determinado grupo, num dado momento de sua trajetória (Gilroy, 2001).

Assim, é possível pensar em modelos de ensino ou propostas pedagógicas nas quais as atividades são o centro irradiador da aprendizagem, contendo em si mesmas as potencialidades para inscrever novos registros de visão de mundo e de compreensão dos fenômenos em estudo e das pessoas em relação. Aí as atividades internas de cada estudante movimentam-se em conexão com seu grupo, levando a focar a própria atividade como unidade de análise. Isto inclui tanto o indivíduo como o grupo com seus ambientes culturalmente definidos (Cole, 1996).

Desse modo, podemos destacar três princípios básicos da teoria da aprendizagem centrada na atividade com auxílio de computador: a) que a atividade desenvolve-se mediada por influências culturais; b) que a atividade deve ser observada e analisada, tendo em consideração os vários níveis que a compõem; e c) que a atividade interna (processos de pensamento) ocorre primeiramente no plano social, quer dizer, imersa em determinado contexto que a caracteriza (Cole, 1996). Com essa compreensão, as atividades de aprendizagem não se tornam mais fáceis, mas fundamentalmente mudam a natureza das tarefas a serem desenvolvidas, propiciando a criação de novas atividades antes não contempladas. É isto que redefine o significado da aprendizagem, colocando-a num patamar diferenciado das aprendizagens que são alcançadas mediante outras perspectivas teóricas. Em vez de entender aprendizagem como uma abstração racional de representações mentais que decorrem das experiências vividas, a aprendizagem é re-conceitualizada, como aprender a participar de acordo com as práticas culturais que guardam pontos de contato, possibilitando visões similares da realidade, comunicando-se entre si, atravessadas por um fundo comum que vai se constituindo pelas experiências dos participantes. Isto supõe aceitar que a participação de um é mediada pela participação dos outros, talvez mais capazes e mais sensíveis para decodificar a cultura que os institui como sujeitos da experiência (Vygotsky, 1998; Hutchins, 1995).

\section{Níveis de Análise}

Uma atividade ou várias atividades que se conjugam numa temática ou foco comum podem ser analisadas em pelo menos três níveis: a) infra-estrutura - condições materiais e equipamentos disponíveis; b) propósitos e intenções do grupo - interesses, motivações e objetivos; e c) pensamento lógico, raciocínio e cognições - idéias, interpretações, críticas e sugestões. 
É preciso, contudo, ter presente as dimensões afetivas, pois elas implicam uma ligação profunda com a atividade e uma relação generosa e dedicada com aquilo que se está fazendo em prol de um objetivo ou projeto (Faria, Marques \& Colla, 2003, p. 238). É isto que sustenta o desejo de persistir nos esforços de interatividade, seja em comunicação eletrônica (e-mails), seja na persistência de constantes consultas aos links, sugeridos pelos textos principais, bem como as buscas reiteradas no imenso cabedal de conhecimentos virtuais disponibilizados na Internet.

Entretanto, o fundamental na teoria da aprendizagem por atividades é que elas são sempre mediadas culturalmente. Isso muda substancialmente a natureza da aprendizagem, ensejando outras atividades que não estavam previstas no planejamento inicial. O ponto crítico é o entendimento da aprendizagem não como uma abstração racional que se traduz em representações mentais, mas como uma mudança fundamental, a partir da experiência que leva à compreensão da aprendizagem como uma prática cultural que se incorpora nos comportamentos, valores e atitudes, movendo as pessoas para um engajamento mais comprometido com determinadas visões de mundo, excluindo ou abandonando posicionamentos anteriores (Gifford \& Enyedy, 1999). Portanto, o básico é a perspectiva do contexto em que as atividades se realizam, bem como o reconhecimento das mediações que estabelecem os matizes de significado dos conteúdos e processos.

\section{Figura 2 - Interatividade e Níveis de Análise.}

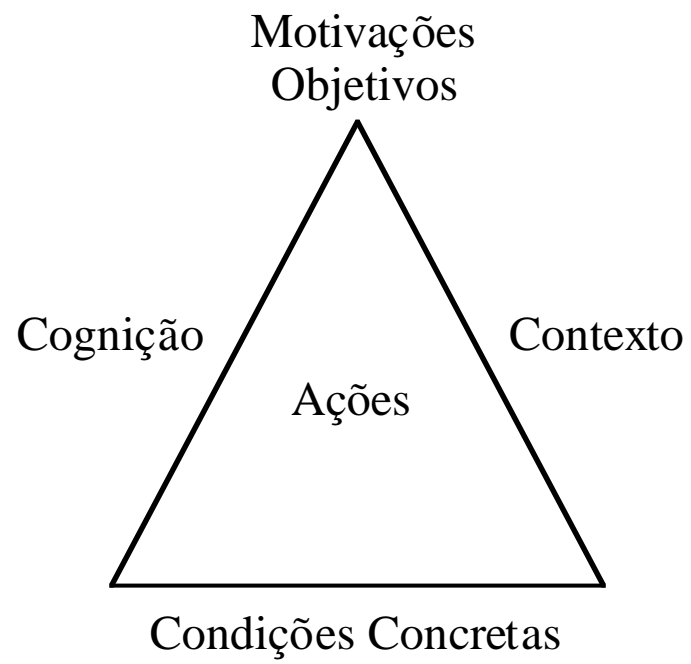

Ao analisar as atividades em diferentes níveis, o nível mais alto distingue-se pelas motivações que o organizam ou pelo objetivo para o qual a atividade se destina. Em seguida, a atividade pode ser examinada em termos das várias ações empreendidas para alcançar objetivos mais imediatos. No nível básico, busca-se analisar os detalhes, consistindo em recortar operações específicas, com suas condições concretas que se transformam em ações objetivamente observáveis. Esses três níveis de análise contribuem para enriquecer a perspectiva que demonstra a interatividade entre a cognição e condições ensejadas pelo contexto material e social em que a atividade de aprendizagem se realiza (Hutchins, 1995).

O terceiro princípio da aprendizagem centrada na atividade é de que a cognição, bem como os instrumentos de mediação de que ela se utiliza têm sua origem na interação social culturalmente produzidas. Apóia-se na teoria de Vygotsky (1998) segundo a qual as funções psicológicas de alta ordem desenvolvem-se primeiro interpsicologicamente, por meio de relações interpessoais que se estabelecem no convívio social. Depois são incorporadas (transladadas) psicologicamente como funções mentais, características de cada indivíduo. Daí o papel decisivo da interação social e, portanto, da conversação, do diálogo e da interlocução nos processos de aprendizagem. Isto implica, também, que os modos de organizar as atividades da aprendizagem, bem como as cognições que delas decorrem, só podem ser compreendidas examinando o contexto histórico que as instaurou.

$\mathrm{Na}$ aprendizagem colaborativa centrada na atividade, desde o início, na fase de planejamento do modelo de ensino propõe-se como foco o desenho de atividades que ajudem o estudante a desenvolver habilidades de formular propostas, comentários e soluções socialmente formuladas. A atividade é orientada para objetivos que dirigem as ações por meio de possíveis usos relevantes dos materiais disponíveis e estruturas sociais, em suas diferentes configurações, evocadas para responder às demandas impostas pela criatividade do grupo em suas interações.

Portanto, não se trata de utilizar o computador per se, em consultas à Internet, em busca de informações, críticas, revisões e comentánios ou em jogos de simulação para resolver problemas, mesmo quando tais atividades envolvem participação dos grupos e interatividade entre seus parti- 
cipantes (Enyedy, Vahey \& Gifford, 1997). Tudo isso pode ser parte das atividades, mas a ênfase está em promover interações que façam emergir os valores e modos de funcionamento das várias culturas que, quando confrontadas em suas diferenças entre os participantes, trazem novas contribuições, enriquecendo as atividades em desenvolvimento. Eles/elas, como participantes ativos, articulam suas intuições, testando suas idéias sistematicamente, analisando, avaliando e discutindo os resultados, numa constante revisão de conceitos, noções e hipóteses explicativas.

Na maioria das vezes, os estudantes trabalham em pares, mas nem sempre atingem consenso e, então, precisam justificar suas discordâncias, estabelecendo uma discussão que faça sentido, para que um terceiro participante avalie os prós e os contras de suas argumentações, extraindo uma conclusão (empírica) dos resultados da atividade desenvolvida. Isto tudo é comunicado aos membros do grupo de sala de aula para que seja novamente discutido, acrescentando outros ângulos de visão. O problema surge quando um participante concorda com a primeira sugestão explicativa, sem explorar possíveis alternativas e sem refletir sobre implicações e conseqüências da posição assumida. Aí, na melhor das hipóteses, um dos membros questiona e desafia outros modos de enfrentar 0 problema, levando a aprofundar a compreensão em termos de contexto e seus desdobramentos.

A aprendizagem colaborativa acontece quando duas ou mais pessoas, interferindo na fala umas das outras, vão construindo pelo intercâmbio de idéias, opiniões e visões de mundo, novos modos de ver a realidade, a sociedade em seus processos de mudança e a si mesmos em suas inter-relações (Marques, 2004).

Ainda é muito o que nos falta para compreender como as condições materiais das atividades da aprendizagem colaborativa se interpenetram com a estrutura social que emana da cultura e das cognições dos estudantes como mediadoras das atividades desenvolvidas com o suporte de computador. Como a cultura é mediadora da cognição? Como se definem os vários componentes que contribuem para delimitar o contexto? Como os sistemas de ensino são mediadores das interações sociais e das diferentes estruturas de participação num ambiente de aprendizagem livre de imposições? Como são criadas comunidades de aprendizagem que sustentam suas práticas numa teoria que delas emana, sem restringir possibilidades de mudança e de novas visões?

\section{Referências}

Cole, M. (1996). Cultural psychology. Cambridge, MA: Harvard University Press.

Enyedy, N., Vahey, P. and Gifford, B. (1977). Active and Supportive Computer:

Mediated Resources for Student-to-Student Conversations. In: Hall, R, Miyake, N. \&

Enyed, N. (Eds.) Computer Suport for Colaborative Learning. Canada: University of Toronto Press.

Faria, E. T., Marques, J. C. \& Colla, A. L. (2003). Gerenciamento e coordenação de

cursos virtuais como desafios à criação de comunidades de aprendizagem. In: Marilú

Fontoura Medeiros e Elaine Turk Faria (Orgs). Educação à distância: cartografias pulsantes em movimento. Porto Alegre: EDIPUCRS.

Gifford, Bernard R. (1996). On the Changing Educational Technical Landscape. Educom Revie. 31 (4):14-19.

Gifford, Bernard R. \& Enyedy, Noel D. (1999). Activity Centered Design: Toward a

Theoretical Framework for CSCL. In: Hoadley, C. \& Roschelle, J. (Eds.) Proceedings of the Computer Support for Collaborative Learning Conference Stanford University.

Mahwah, N.J.: Lawrence Erlbaum. Gilroy, Kathleen (2001). Collaborative E-Learning: The Right Approach.

W W W.destination c rm . com/articles/ default.asp?ArtclesID=1767.Acesso em 29/06/2005.

Hutchins, E. (1995). Cognition in the Wild. Cambridge, MA: Massachussets Institute of Technology Press.

Lave, J. (1996). Teaching as Learning in Pratice. Mind, Culture and Activity. 3(3), 149-164.

Marques, J. C. (2004). Usos da inteligência para o sucesso: aprendizagem colaborativa e comunida- 
des de aprendizagem. Psico. Porto Alegre, PUCRS. 35(2), 125-132.

Marques, J. C. (2004). O mundo do trabalho e suas complexidades. In: Jorge Castellá

Sarriera et al (orgs.) Desafios do mundo do trabalho: orientação, inserção e mudanças. Porto Alegre: EDIPUCRS.

Schwandt, T. (1999). The study of understanding and its implications for qualitative inquiry. Qualitative Inquiry. 5(4), 451-464.
Sternberg, R. (1985). Beyond IQ: A triarchic theory of human intelligence. New York: Cambridge University Press.

Vygotsky, L (1998). Mind in Society. In: M. Cole, V. John Steiner, S. Scribner and E.

Souberman (Eds.) The Development of Higher Order Psychological Processes. Cambridge, MA: Harvard Univesity Press.

Recebido em/received in: 29/07/2005

Aprovado em/approved in: 25/08/2005 